\title{
Overexpression of TRIM44 is an independent marker for predicting poor prognosis in epithelial ovarian cancer
}

\author{
SHUANG LIU*, HEXUAN YIN*, HONGYING JI, JIAQI ZHU and RONG MA \\ Department of Gynaecology and Obstetrics, Harbin Medical University \\ Cancer Hospital, Harbin, Heilongjiang 150081, P.R. China
}

Received February 8, 2018; Accepted June 29, 2018

DOI: $10.3892 /$ etm.2018.6541

\begin{abstract}
Tripartite motif-containing 44 (TRIM44) has been demonstrated to be important in tumor metastasis and progression. However, the expression pattern and prognostic value of the expression of TRIM44 in epithelial ovarian cancer (EOC) remain to be fully elucidated. In the present study, the aim was to investigate the expression and clinical role of TRIM44 in EOC. A total of 109 patients, who underwent primary surgery with the goal of maximal tumor resection followed by standard combination chemotherapy with carboplatin and paclitaxel, were analyzed in the present study. The expression level of TRIM44 was determined by western blot analysis and immunohistochemistry in 109 ECO tissues. It was found that the expression of TRIM44 was low in normal tissues and high in EOC tissues. Univariate survival analysis showed that the overexpression of TRIM44 was significantly associated with International Federation of Gynecology and Obstetrics stage and lymph node metastasis $(\mathrm{P}<0.05)$. Kaplan-Meier analysis suggested that there was a significant difference in overall survival and disease-free survival rates between patients with a high expression of TRIM44 and patients with a low expression of TRIM44. Patients with a high expression level of TRIM44 exhibited poorer overall survival and disease-free survival rates, compared with patients expressing a low level of TRIM44 $(\mathrm{P}<0.001)$. In addition, the results of the multivariate analysis revealed that the predictive value of the expression of TRIM44 was independent of other clinicopathological factors for predicting prognosis. These
\end{abstract}

Correspondence to: Dr Rong Ma, Department of Gynaecology and Obstetrics, Harbin Medical University Cancer Hospital, 150 Haping Road, Nangang, Harbin, Heilongjiang 150081, P.R. China

E-mail: dr_marong2017@126.com

*Contributed equally

Abbreviations: TRIM44, tripartite motif-containing 44; EOC, epithelial ovarian cancer; FIGO, International Federation of Gynecology and Obstetrics

Key words: tripartite motif-containing protein 44, epithelial ovarian cancer, metastasis, prognosis findings demonstrated that a high expression of TRIM44 was associated with the progression and prognosis of EOC.

\section{Introduction}

Epithelial ovarian cancer (EOC) is the most common cause of mortality among women with gynecological malignancies. The majority of patients with EOC are diagnosed at an advanced clinical stage, as they are asymptomatic until the tumor has metastasized on the surface of the peritoneum. Based on GLOBOCAN estimates, there were 238,700 new cases of ovarian cancer and 151,900 cases of ovarian cancer-associated mortality worldwide in 2012 (1). Despite surgery combined with chemotherapy as the main strategy for treatment, chemoresistance exists in $25 \%$ of patients, and the majority ultimately experience relapse (2). Therefore, it is important to identify a potential novel therapeutic target for patients with EOC.

Tripartite motif-containing protein 44 (TRIM44) belongs to the tripartite motif protein family, which comprises structurally-related proteins and are involved in multiple cellular processes, including cell proliferation, migration, apoptosis and cancer cell invasion. Studies have suggested that TRIM44 may have an oncogenic role in the invasion and metastases of several types of human cancer, including prostate cancer (3), hepatocellular carcinoma (4), gastric carcinoma (5), non-small cell lung cancer (6) and testicular germ cell tumors (7). However, at present, the precise function of TRIM44 and its underlying carcinogenic mechanism in ovarian cancer remain to be fully elucidated.

Therefore, the present study aimed to investigate the clinical significance of the expression of TRIM44 in patients with EOC, and performed further experiments to determine whether TRIM44 may be a potential biomarker for patients with EOC.

\section{Materials and methods}

Ethics statement. The present study was approved by the Ethical Committee of the Harbin Medical University Cancer Hospital (Harbin, China).

Patient population. In total, 109 patients underwent primary surgery with the goal of maximal tumor resection followed by standard combination chemotherapy with carboplatin and paclitaxel between March 2010 and March 2013. None of the patients had received neoadjuvant chemotherapy and/or 
radiation prior to surgery. All cases were diagnosed according to the Silverberg grading system (8) and the International Federation of Gynecology and Obstetrics (FIGO) staging system. The patients' characteristics, including age, FIGO stage, histological grade, histological type, and lymph node metastasis, are summarized in Table I. Normal samples in the present study were obtained from women undergoing ovariectomy for benign gynecologic disease at the Department of Gynecology of the Harbin Medical University Cancer Hospital.

Western blot analysis. The protein expression levels were evaluated by western blot analysis with an anti-TRIM44 antibody. Total proteins from nine frozen tissue samples were extracted using RIPA buffer (Beyotime Institute of Biotechnology, Haimen, China). Protein concentration was determined by a Bradford assay using bovine serum albumin (Shanghai Ruisai Biotechnology Co., Ltd., Shanghai, China). Proteins (50 $\mu \mathrm{g} /$ lane) were electrophoretically separated on 5\% sodium dodecyl sulfate-polyacrylamide gels and transferred onto polyvinylidene difluoride membranes (EMD Millipore, Billerica, MA, USA). The membranes were blocked in 5\% fat-free milk at room temperature for $1 \mathrm{~h}$. Primary antibodies against TRIM44 (cat. no. NBP2-31681; 1:300; Novus Biologicals, LLC, Littleton, CO, USA) and $\beta$-actin (cat. no. WL01845; 1:1,000; Wanleibio Co., Ltd., Harbin, China) were diluted in buffer and incubated with the samples at $4^{\circ} \mathrm{C}$ overnight. The membranes were then incubated with secondary antibodies from and visualized with reagents from the Phototope ${ }^{\circledR}$-HRP Western Blot Detection System (cat. no. 7071; Cell Signaling Technology, Inc., Danvers, MA, USA). The experiment was performed in triplicate.

Immunohistochemistry and evaluation. Immunohistochemistry was performed using an anti-TRIM44 antibody following standard methods. The tissue specimens were paraffin-embedded and tissue blocks were cut into $5-\mu \mathrm{m}$-thick sections. Following deparaffinization and rehydration, the sections were incubated in $0.3 \%$ hydrogen peroxide at room temperature. Blocking with peroxidase was performed for 10 min. TRIM44 antigen retrieval was performed by heating the sections in a stainless autoclave. Following washing in PBS, the sections were incubated with the anti-TRIM44 antibody (Abcam, Cambridge, UK) at a dilution of 1:100, and then placed into a humidor overnight at $4^{\circ} \mathrm{C}$. The slides were counterstained with hematoxylin for $2 \mathrm{~min}$.

According to the number of positive tumor cells, the staining was scored as follows: ' 0 ' if $<10 \%$ of the tumor cells were positively stained, ' 1 ' if $10-33 \%$, and ' 2 ' if $34-66 \%$, ' 3 ' if $67-100 \%$. The staining intensity was scored as follows: Score 0 (negative), score 1 (weak) and score 2 (moderate) in at least five different high-power fields with a light microscope. The protein expression levels of TRIM44 were classified semi-quantitatively based on the total combined score of the positive-staining tumor cell percentage and the staining intensity. The sum of the staining intensity score and the percentage of positive staining was used to evaluate expression levels, where $<3$ indicated low expression and $\geq 3$ indicated high expression (5).

The immunohistochemistry scoring procedure was performed twice by two independent pathologists who were experienced in assessing immunohistochemistry and had no knowledge of the clinicopathological information of the slides.
Statistical analyses. Pearson's $\chi^{2}$ square test was used for determining proportional differences in univariate analyses. The survival curve was generated using the Kaplan-Meier and the log-rank test. The logistic regression model was used for univariate and multivariate analyses. All tests were two-sided and $\mathrm{P} \leq 0.05$ was considered to indicate a statistically significant difference. Statistical analysis was performed using SPSS 21.0 statistical software (IBM SPSS, Armonk, NY, USA).

\section{Results}

Clinicopathologicalfeatures of patients with EOC. The present study analyzed a total of 109 patients, who underwent primary surgery with the goal of maximal tumor resection followed by standard combination chemotherapy with carboplatin and paclitaxel. The clinicopathological features of the patients enrolled in the study are summarized in Table I. Among them, $12(11 \%)$ patients were diagnosed with grade 1 (G1) EOC and 97 patients $(89 \%)$ were diagnosed with grade 2 and grade 3 (G2/G3) EOC. According to the American Joint Committee on Cancer (AJCC) tumor staging criteria, eight (7.3\%) patients were stage I, 23 (21.1\%) patients were stage II, 75 (68.8\%) patients were stage III, and three $(2.8 \%)$ patients were stage IV. There were $26(23.9 \%)$ patients with distant metastases at the time of diagnosis. The 109 patients were further grouped into four histological types, which included 92 patients with serous type, seven patients with mucinous type, five patients with endometrioid type and five patients with the clear cell type. The survival analysis of all patients with EOC was followed up until 11 January 2017. The mean follow-up duration was 36.28 months (range, 3-77 months).

Correlation between the expression levels of TRIM44 and the clinicopathological characteristics of EOC. The protein expression of TRIM44 in EOC and normal ovarian tissues was measured by western blot analysis. As shown in Fig. 1, the protein expression of TRIM44 in EOC tissues was significantly higher than that in normal tissues $(\mathrm{P}<0.001$; Fig. $1 \mathrm{~A}$ and $\mathrm{B})$. The expression of TRIM44 was further detected using immunohistochemistry. The results of the immunohistochemical staining are shown in Fig. 2A-E. A high level of immunoreactivity for TRIM44 was found in the plasma of EOC tissues. As shown in Table I, there was significant correlation between the expression of TRIM44 and AJCC stage $(\mathrm{P}<0.001)$, histological grade $(\mathrm{P}=0.053)$, histological type $(\mathrm{P}<0.001)$ and lymph node metastasis $(\mathrm{P}=0.015)$. Furthermore, the positive expression rate of TRIM44 in patients with late-stage (stage III and IV) disease was $92.3 \%$ (72/78), which was significantly higher than the rate in patients with early-stage (stage I and II) disease of $67.7 \%(21 / 31)(\mathrm{P}<0.001)$. Patients with serous type $(91.3 \%$, 84/92) tended to express higher levels of TRIM44, compared with the other three histological types (mucinous, 42.9\%, 3/7; endometrioid, 60\%, 3/5; and clear cell, 60\%, 3/5) $(<0.001)$. In addition, the expression level of TRIM44 in patients with lymph node metastasis $(100 \%, 26 / 26)$ was significantly higher, compared with the level in patients without lymph node metastasis $(80.7 \%, 67 / 83)(\mathrm{P}=0.015)$. The positive expression rate of TRIM44 in patients with moderately or poorly differentiated disease (grade G2/3; 87.6\%, 85/97) was significantly higher than that in patients with well-differentiated disease 
Table I. Association analyses between the expression levels of TRIM44 and the clinicopathological characteristics of epithelial ovarian cancer.

TRIM44 expression

\begin{tabular}{|c|c|c|c|c|}
\hline & & & & \\
\hline Variable & Patients (n) & Low, n (\%) & High, n (\%) & P-value \\
\hline Age (years) & & & & 0.097 \\
\hline$\leq 55$ & 61 & $12(19.7)$ & $49(80.3)$ & \\
\hline$>55$ & 48 & $4(8.3)$ & $44(91.7)$ & \\
\hline FIGO stage & & & & $<0.001$ \\
\hline I & 8 & $8(100.0)$ & $0(0.0)$ & \\
\hline II & 23 & $2(8.7)$ & $21(91.3)$ & \\
\hline III & 75 & $6(8.0)$ & $69(92.0)$ & \\
\hline IV & 3 & $0(0.0)$ & $3(100.0)$ & \\
\hline Histological grade & & & & 0.053 \\
\hline G1 & 12 & $4(33.3)$ & $8(66.7)$ & \\
\hline $\mathrm{G} 2 / \mathrm{G} 3$ & 97 & $12(12.4)$ & $85(87.6)$ & \\
\hline Histological type & & & & $<0.001$ \\
\hline Serous & 92 & $8(8.7)$ & $84(91.3)$ & \\
\hline Mucinous & 7 & $4(57.1)$ & $3(42.9)$ & \\
\hline Endometrioid & 5 & $2(40.0)$ & $3(60.0)$ & \\
\hline Clear cell & 5 & $2(40.0)$ & $3(60.0)$ & \\
\hline Lymph node metastasis & & & & 0.015 \\
\hline No & 83 & $16(19.3)$ & $67(80.7)$ & \\
\hline Yes & 26 & $0(0.0)$ & $26(100.0)$ & \\
\hline
\end{tabular}

${ }^{\mathrm{a}} \chi^{2}$ test. TRIM44, tripartite motif-containing protein 44; FIGO, International Federation of Gynecology and Obstetrics; G1, well differentiated; G2, moderately differentiated; G3, poorly differentiated.
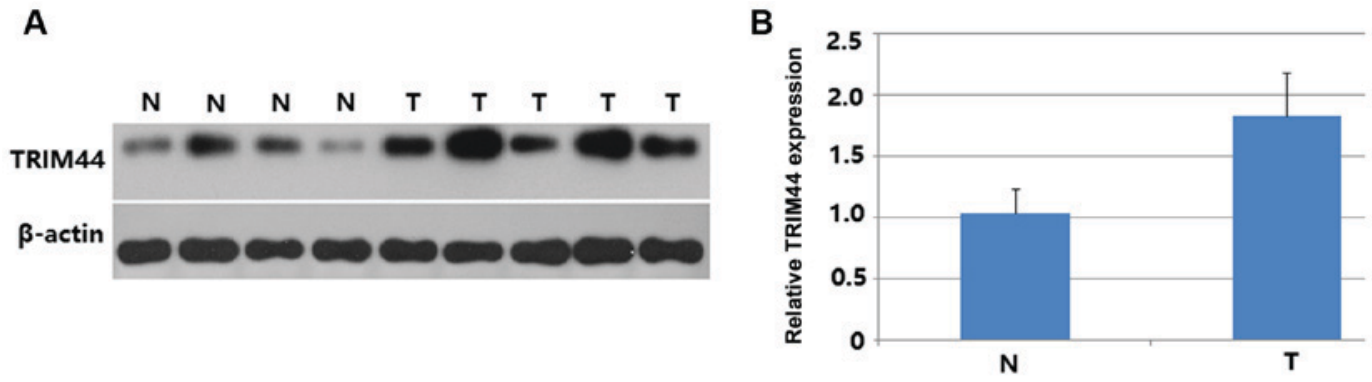

Figure 1. Protein expression of TRIM44 in EOC tissues. (A) Protein samples obtained from frozen $\mathrm{N}$ and T ovarian tissues were analyzed by western blot analysis. Levels of $\beta$-actin were used as an internal control. (B) Histogram of pooled data from $N(n=4)$ and $T(n=5)$ samples. Expression of TRIM44 was elevated in the $\mathrm{T}$ samples, compared with the $\mathrm{N}$ samples $(\mathrm{P}<0.001)$. The data are presented as the mean \pm standard deviation. TRIM44, tripartite motif-containing 44; $\mathrm{N}$, normal ovarian tissue; $\mathrm{T}$, epithelial ovarian cancer tissue.

(grade $\mathrm{G} 1 ; 66.7 \%, 8 / 12)(\mathrm{P}=0.053)$. No significant correlation existed between the expression of TRIM44 and patient age $(\mathrm{P}=0.097)$. The results of the immunohistochemical analysis of the expression of TRIM44 in EOC are summarized in Table I.

Prognostic value of the expression of TRIM44 in patients with $E O C$. To investigate the prognostic value of the expression of TRIM44 in patients with EOC, Kaplan-Meier analysis and univariate survival analysis of overall survival and disease-free survival rates were performed for the 109 patients with EOC. The 109 patients with EOC were firstly classified into two patient subgroups: Patients with a high expression of TRIM44 $(n=93)$ and patients with a low expression of TRIM44 $(n=16)$ according to the expression levels of TRIM44. The Kaplan-Meier analysis showed that overall survival rate between patients with a high expression of TRIM44 and patients with a low expression of TRIM44 was significantly different ( $\mathrm{P}=0.0002$; Fig. 3). Patients expressing a high level of TRIM44 exhibited poorer overall survival rate than patients expressing a low level of TRIM44 ( $\mathrm{P}<0.001$; Table II). In addition, the difference in disease-free survival rate between patients with a high expression of TRIM44 and patients with a low expression of TRIM44 was also significantly different $(\mathrm{P}<0.0001$; Fig. 4). Patients with a high level of TRIM44 had 
Table II. Univariate survival analysis of OS and DFS rates in 109 patients with epithelial ovarian cancer.

\begin{tabular}{|c|c|c|c|c|c|c|c|}
\hline \multirow[b]{2}{*}{ Variable } & \multirow[b]{2}{*}{$\mathrm{n}$} & \multicolumn{2}{|c|}{ OS } & \multirow[b]{2}{*}{ P-value ${ }^{b}$} & \multicolumn{2}{|c|}{ DFS } & \multirow[b]{2}{*}{ P-value ${ }^{b}$} \\
\hline & & Months $^{\mathrm{a}}$ & $95 \% \mathrm{CI}$ & & Months $^{\mathrm{a}}$ & $95 \% \mathrm{CI}$ & \\
\hline Age (years) & & & & 0.130 & & & 0.028 \\
\hline$\leq 55$ & 61 & $43 \pm 3$ & $37-49$ & & $32 \pm 3$ & $27-38$ & \\
\hline$>55$ & 48 & $34 \pm 3$ & $27-41$ & & $22 \pm 3$ & $17-27$ & \\
\hline FIGO stage & & & & $<0.001$ & & & $<0.001$ \\
\hline I & 8 & $57 \pm 10$ & $38-76$ & & $58 \pm 10$ & $39-76$ & \\
\hline II & 23 & $48 \pm 5$ & $38-58$ & & $35 \pm 5$ & $27-44$ & \\
\hline III & 75 & $35 \pm 2$ & $30-40$ & & $23 \pm 2$ & $19-26$ & \\
\hline IV & 3 & $15 \pm 5$ & $5-25$ & & $11 \pm 5$ & $2-20$ & \\
\hline Histological grade & & & & 0.108 & & & 0.102 \\
\hline G1 & 12 & $49 \pm 6$ & $36-61$ & & $36 \pm 6$ & $24-48$ & \\
\hline $\mathrm{G} 2 / \mathrm{G} 3$ & 97 & $38 \pm 2$ & $33-42$ & & $27 \pm 2$ & $22-31$ & \\
\hline Histological type & & & & 0.697 & & & 0.200 \\
\hline Serous & 92 & $38 \pm 2$ & $34-43$ & & $26 \pm 2$ & $22-30$ & \\
\hline Mucinous & 7 & $36 \pm 10$ & $17-56$ & & $32 \pm 10$ & $12-52$ & \\
\hline Endometrioid & 5 & $40 \pm 9$ & $24-57$ & & $37 \pm 11$ & $16-58$ & \\
\hline Clear cell & 5 & $45 \pm 11$ & $23-67$ & & $45 \pm 11$ & $22-67$ & \\
\hline Lymph node metastasis & & & & 0.002 & & & 0.014 \\
\hline No & 83 & $41 \pm 3$ & $35-46$ & & $30 \pm 3$ & $25-36$ & \\
\hline Yes & 26 & $33 \pm 3$ & $27-39$ & & $20 \pm 2$ & $15-24$ & \\
\hline TRIM44 & & & & $<0.001$ & & & $<0.001$ \\
\hline Low expression & 16 & $60 \pm 6$ & $49-72$ & & $56 \pm 6$ & $43-69$ & \\
\hline High expression & 93 & $36 \pm 2$ & $31-40$ & & $23 \pm 2$ & $20-27$ & \\
\hline
\end{tabular}

${ }^{a}$ Values are presented as the mean \pm standard error of the mean; ${ }^{b}$ Log-rank test. FIGO, International Federation of Gynecology and Obstetrics; G1, well differentiated; G2, moderately differentiated; G3, poorly differentiated; TRIM44, tripartite motif-containing protein 44; OS, overall survival; DFS, disease-free survival; CI, confidence interval.

A

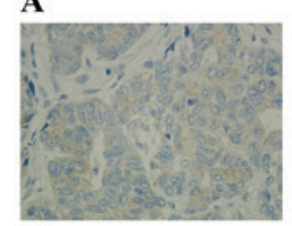

B

C

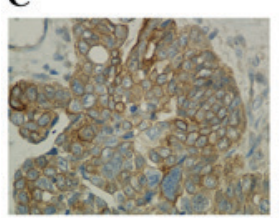

$\mathbf{E}$

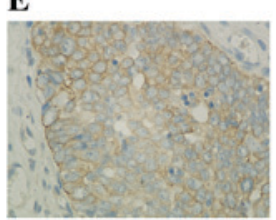

Figure 2. Immunohistochemical staining of TRIM44 in epithelial ovarian cancer specimens. Magnification, x400. (A and B) Low expression of TRIM44 in epithelial ovarian cancer with scores of 2. (C and D) High expression of TRIM44 in epithelial ovarian cancer with scores of 4. (E) High expression of TRIM44 in epithelial ovarian cancer with a score of 3. TRIM44, tripartite motif-containing 44. a shorter disease-free survival rate than patients with a low expression of TRIM44 $(\mathrm{P}<0.001$; Table II).

Independent prognostic value of the expression of TRIM44. The present study performed multivariate Cox regression analysis to examine whether the predicative value of the expression of TRIM44 is independent of tumor stage in predicting prognosis in EOC. The multivariate analysis showed that a high expression of TRIM44 was an independent prognostic factor associated with overall survival rate $(\mathrm{HR}=3.662,95 \%$ CI 1.313-10.215, $\mathrm{P}=0.013$ ) and disease-free survival rate $(\mathrm{HR}=4.021,95 \%$ CI $1.586-10.192, \mathrm{P}=0.003)$, respectively (Table III). Therefore, these results indicated that the predictive value of the expression of TRIM44 was independent of other clinicopathological factors.

\section{Discussion}

In the present study, the expression of TRIM44 in 109 EOC specimens was investigated using immunohistochemistry of the protein levels. To the best of our knowledge, the present study provides one of the most comprehensive reports on the correlation between the expression of TRIM44 and the 
Table III. Multivariate analysis of overall survival and disease-free survival rates in 109 patients with epithelial ovarian cancer.

\begin{tabular}{lccccc}
\hline Variable & HR & $95 \%$ CI & P-value $^{\mathrm{a}}$ & HR & 95\% CI \\
\hline FIGO stage & 1.858 & $1.165-2.963$ & 0.009 & 1.835 & $1.187-2.836$ \\
TRIM44 & 3.662 & $1.313-10.215$ & 0.013 & 4.021 & $1.586-10.192$ \\
\hline
\end{tabular}

${ }^{\mathrm{a} C o x}$ regression analysis. FIGO, International Federation of Gynecology and Obstetrics; TRIM44, tripartite motif-containing protein 44; HR, hazard ratio; CI, confidence interval.

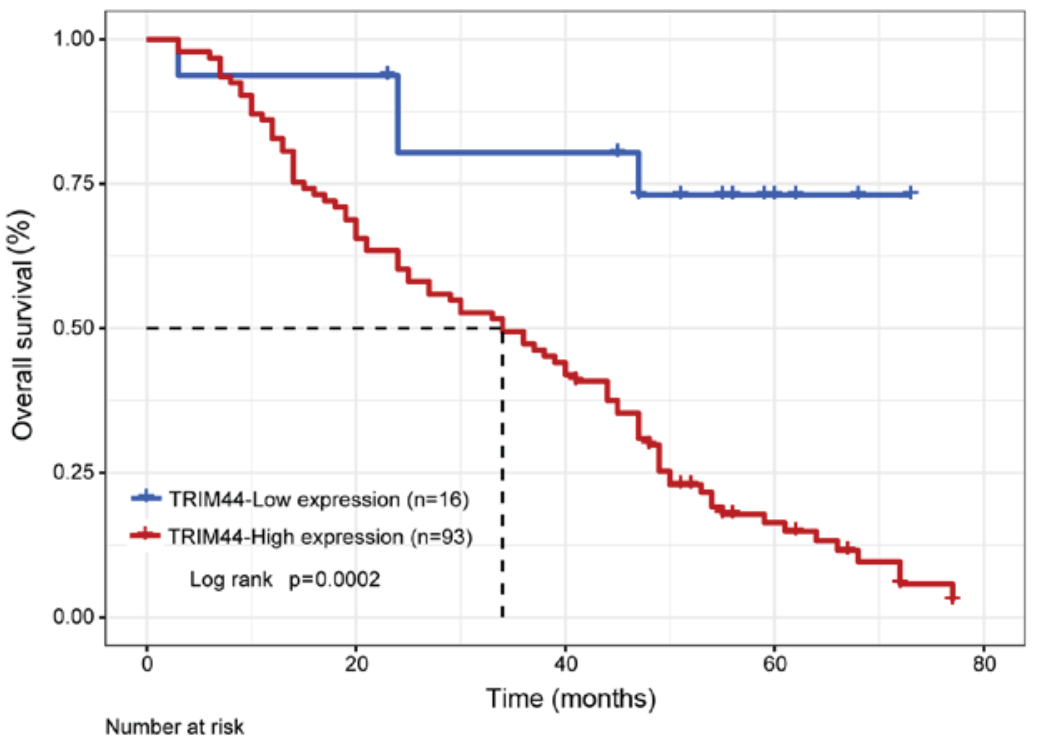

\begin{tabular}{l|ccccc|}
\cline { 2 - 5 } TRIM44-Low expression & 16 & 15 & 12 & 4 & 0 \\
\hline TRIM44-High expression & 93 & 64 & 41 & 11 & 0 \\
\cline { 2 - 6 } & & & & &
\end{tabular}

Figure 3. Kaplan-Meier analysis of overall survival rate relative to the expression of TRIM44. Patients with a high expression of TRIM44 had a poorer overall survival rates than those of patients with a low expression of TRIM44. TRIM44, tripartite motif-containing 44.

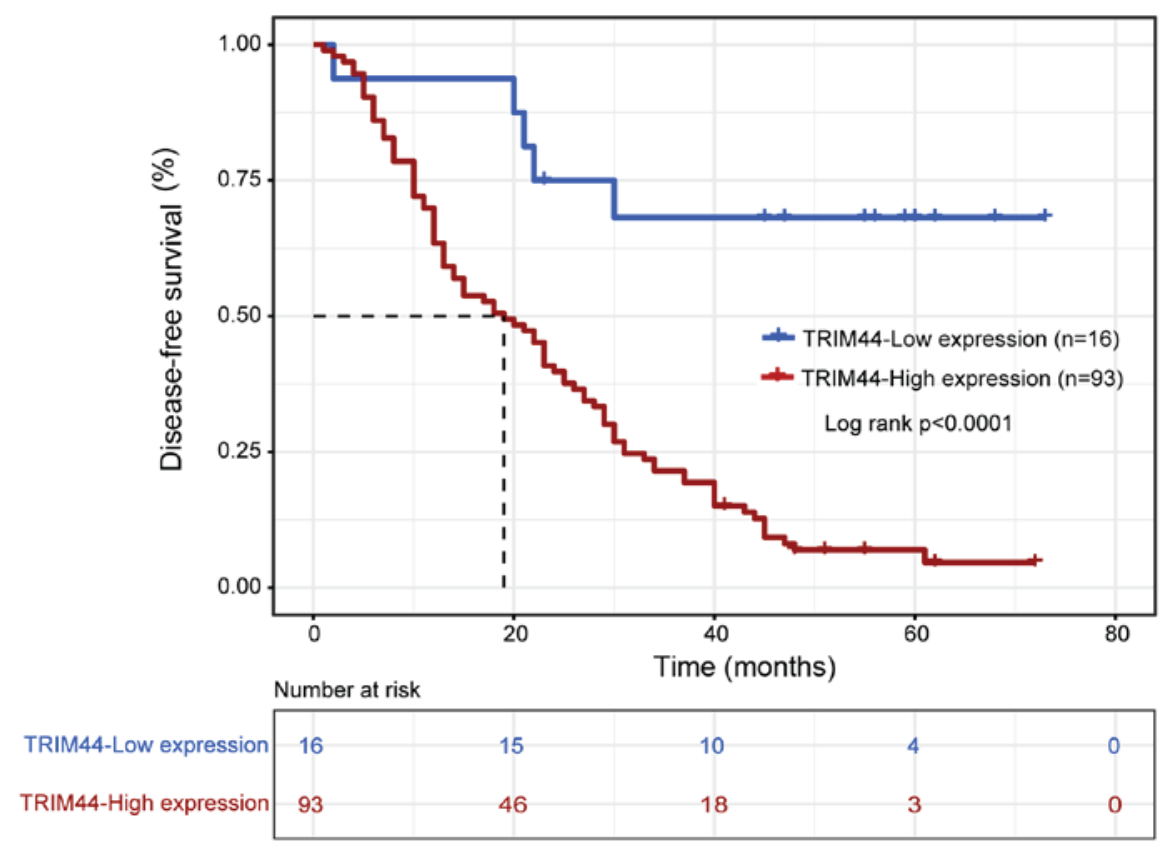

Figure 4. Kaplan-Meier analysis of disease-free survival rate relative to the expression of TRIM44. Patients with a high expression of TRIM44 had a poorer disease-free survival rates than those of patients with a low expression of TRIM44. TRIM44, tripartite motif-containing 44. 
clinicopathological features of EOC in the literature. The results of the present study indicated that the expression of TRIM44 was significantly higher in cases assigned a high FIGO stage $(\mathrm{P}<0.001)$. The expression of TRIM44 was also significantly increased in patients with cancer exhibiting lymph node metastasis $(\mathrm{P}=0.015)$. To examine the association between the expression of TRIM44 and patient prognosis, the present study first analyzed the protein expression of TRIM44 in EOC and found a prognostic correlation. The data demonstrated that patients with a high expression of TRIM44 had significantly poorer overall survival and disease-free survival rates, compared with patients with a low expression of TRIM44. A multivariate analysis showed that the expression of TRIM44 was an independent prognostic factor for overall survival and disease-free survival rates in patients with EOC. Consistent with previous reports examining the role of TRIM44 in the progression of other tumor types, the group with a high protein expression of TRIM44 also exhibited a poor prognosis $(6,7,9)$.

Previous reports have investigated the pathogenesis by which TRIM44 promotes cancer development. A previous study indicated that a high expression of TRIM44 was associated with poor prognosis and that TRIM44 may be involved in cell proliferation, migration, and anti-apoptotic effects in testicular germ cell tumors (7). Zhu et al (4) demonstrated that the overexpression of TRIM44 enhanced the invasive and migratory capacities of hepatocellular carcinoma cells. Kashimoto et al (5) suggested that TRIM44 may be crucial in tumor cell proliferation through its overexpression, and indicated its usefulness as a predictor and potential therapeutic target in gastric cancer.

Several studies have demonstrated that TRIM44 is involved in cancer progression and metastasis. A previous study demonstrated that the silencing of TRIM44 may inhibit the proliferation, migration and invasion of human papillary thyroid cancer cells through suppression of the Wnt/ $\beta$-catenin signaling pathway (10). Kawabata et al demonstrated that TRIM44 knockdown attenuated the tumor necrosis factor- $\alpha$-dependent phosphorylation of the p65 subunit of nuclear factor (NF) $-\kappa B$ and inhibitor of $\mathrm{NF}-\kappa \mathrm{B} \alpha$ in breast cancer cells (11). Another study inferred that the knockdown of TRIM44 inhibited the proliferation and invasion of prostate cancer cells through inactivation of the phosphoinositide 3-kinase/Akt signaling pathway (3). A study in China found that TRIM44 may promote proliferation and metastasis in non-small cell lung cancer via the mammalian target of rapamycin signaling pathway (6).

The present study was limited to several known clinicopathological factors that were examined for association with the protein expression of TRIM44. Further investigations are required to evaluate this expression with other risk factors for EOC.

The present study demonstrated that overexpression of TRIM44 protein can be used as an independent prognostic factor for assessing disease progression and poor prognosis. These results suggest that TRIM44 may be an attractive therapeutic target for the treatment of EOC. However, these findings require confirmation in a larger study.

\section{Acknowledgements}

The authors would like to thank Dr Huike Yang (Department of Anatomy, Harbin Medical University) for the statistical analysis.

\section{Funding}

This study was supported by grants of the Abroad Science Foundation of Heilongjiang Province (grant no. LC2012C14). The funders had no role in study design, data collection and analysis, decision to publish, or preparation of the manuscript.

\section{Availability of data and materials}

The datasets used and/or analyzed during the current study are available from the corresponding author on reasonable request.

\section{Authors' contributions}

RM conceived and designed the experiments; SL, HY, HJ and JZ performed the experiments and analyzed the data; RM wrote the manuscript. All authors have read and approved the final manuscript.

\section{Ethics approval and consent to participate}

The present study was completed in compliance with the Helsinki Declaration and was approved by the Ethical Committee of the Harbin Medical University Cancer Hospital. The data collection and analysis were performed without disclosing patients' identities.

\section{Patient consent for publication}

Not applicable.

\section{Competing interests}

The authors declare that they have no competing interests.

\section{References}

1. Torre LA, Bray F, Siegel RL, Ferlay J, Lortet-Tieulent J and Jemal A: Global cancer statistics, 2012. CA Cancer J Clin 65: 87-108, 2015.

2. Siegel RL, Miller KD and Jemal A: Cancer statistics, 2015. CA Cancer J Clin 65: 5-29, 2015.

3. Tan Y, Yao H, Hu J and Liu L: Knockdown of TRIM44 inhibits the proliferation and invasion in prostate cancer cells. Oncol Res 25: 1253-1259, 2017.

4. Zhu X, Wu Y, Miao X, Li C, Yin H, Yang S, Lu X, Liu Y, Chen Y, Shen R, et al: High expression of TRIM44 is associated with enhanced cell proliferation, migration, invasion, and resistance to doxorubicin in hepatocellular carcinoma. Tumour Biol 37: 14615-14628, 2016.

5. Kashimoto K, Komatsu S, Ichikawa D, Arita T, Konishi H, Nagata H, Takeshita H, Nishimura Y, Hirajima S, Kawaguchi T, et al: Overexpression of TRIM44 contributes to malignant outcome in gastric carcinoma. Cancer Sci 103: 2021-2026, 2012.

6. Xing Y, Meng Q, Chen X, Zhao Y, Liu W, Hu J, Xue F, Wang X and Cai L: TRIM44 promotes proliferation and metastasis in non-small cell lung cancer via mTOR signaling pathway. Oncotarget 7: 30479-30491, 2016.

7. Yamada Y, Takayama KI, Fujimura T, Ashikari D, Obinata D, Takahashi S, Ikeda K, Kakutani S, Urano T, Fukuhara H, et al: A novel prognostic factor TRIM44 promotes cell proliferation and migration, and inhibits apoptosis in testicular germ cell tumor. Cancer Sci 108: 32-41, 2017.

8. Silverberg SG: Histopathologic grading of ovarian carcinoma: A review and proposal. Int J Gynecol Pathol 19: $7-15,2000$ 
9. Ong CA, Shapiro J, Nason KS, Davison JM, Liu X, Ross-Innes C, O'Donovan M, Dinjens WN, Biermann K, Shannon N, et al: Three-gene immunohistochemical panel adds to clinical staging algorithms to predict prognosis for patients with esophageal adenocarcinoma. J Clin Oncol 31: 1576-1582, 2013.

10. Zhou Z, Liu Y, Ma M and Chang L: Knockdown of TRIM44 inhibits the proliferation and invasion in papillary thyroid cancer cells through suppressing the Wnt $/ \beta$-catenin signaling pathway. Biomed Pharmacother 96: 98-103, 2017.
11. Kawabata H, Azuma K, Ikeda K, Sugitani I, Kinowaki K, Fujii T, Osaki A, Saeki T, Horie-Inoue K and Inoue S: TRIM44 is a poor prognostic factor for breast cancer patients as a modulator of NF-кB signaling. Int J Mol Sci 18: E1931, 2017.

(c) (1) $(9)$ This work is licensed under a Creative Commons EY No No Attribution-NonCommercial-NoDerivatives 4.0 International (CC BY-NC-ND 4.0) License. 